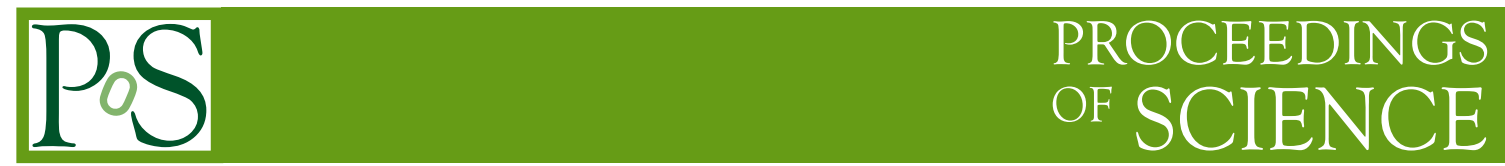

\title{
Missing ET and jets, trigger and reconstruction efficiency
}

Matti KORTELAINEN* on behalf of the CMS Collaboration

Helsinki Institute of Physics, Finland

E-mail: matti.kortelainen@helsinki.fi

The reconstruction of the missing transverse energy and jets, the trigger plans and the reconstruction efficiencies in the CMS detector are discussed. The performance with the $7 \mathrm{TeV}$ protonproton collision data is presented.

Prospects for Charged Higgs Discovery at Colliders

27-30 September 2010

Uppsala University, Sweden

* Speaker. 


\section{Introduction}

Jets and missing transverse energy are important signatures in various charged Higgs boson decay channels. A good understanding of them is also important for the many Standard Model background processes. In this note the performance of jet and missing $E_{T}$ reconstruction in the CMS detector at LHC from the early $7 \mathrm{TeV} p p$ collisions are presented. CMS has a superconducting solenoid, of $6 \mathrm{~m}$ internal diameter, providing a field of $3.8 \mathrm{~T}$. Within the field volume are the silicon pixel and strip tracker, the crystal electromagnetic calorimeter (ECAL) and the brass/scintillator hadron calorimeter (HCAL). Muons are measured in gas-ionization detectors embedded in the steel return yoke. The detailed description of the CMS detector is given in Ref. [1].

Four types of jets are reconstructed at CMS, which differently combine individual contributions from subdetectors to the jet clustering algorithm. Calorimeter jets [2] are reconstructed using the energy deposits in ECAL and HCAL cells, combined into calorimeter towers. The Jet-plusTracks algorithm (JPT) [3] adds the momenta of charged particle tracks associated to the previously reconstructed calorimeter jets to the jet energy, and subtracts the expected average energy depositions of the tracks whose projections onto the calorimeter surface point to within the jet cone around the jet axis. The Particle Flow (PFlow or PF) jets are reconstructed from the list of particles provided by the complete Particle Flow algorithm [4, 5], which combines the information from all CMS subdetectors to identify and reconstruct all particles in the event. Track jets [6] are reconstructed from the tracks of the charged particles providing a method which is completely independent from the calorimetric measurements, allowing for cross-checks. Here the emphasis is on the former three types of jets. Jets in the studies presented here are reconstructed using the Anti- $k_{T}$ [7] clustering algorithm with the size parameter $R=0.5$.

Missing transverse energy $\left(\mathbb{E}_{T}\right)$ is generally calculated as the magnitude of the negative vector sum of the momentum transverse to the beam axis of all final-state particles reconstructed in the detector. The most traditional and common algorithm uses energies deposited in calorimeter towers and assumes mass-less objects based on energies measured in the tower and angles defined by a vector from the reconstructed primary vertex to the tower. CMS has implemented four major types of algorithms to reconstruct $\mathbb{E}_{T}$. Calorimeter $\mathbb{E}_{T}$ [8] is based on the calorimeter energies as described above. Track-corrected $\mathbb{E}_{T}$ [9] is calculated by replacing the calorimeter tower energies matched to charged hadrons with their corresponding charged track momenta. Particle Flow $\mathbb{E}_{T}$ [4] is calculated using the complete Particle Flow technique. $\mathbb{E}_{T}$ can also be calculated using the reconstructed jets $\left(H_{T}\right)$. Here the focus is on the former three types of $\mathbb{E}_{T}$.

The note is organized as follows. The jet energy calibration and the estimation of $p_{T}$ resolution from the collision data are presented in Section 2. A data versus Monte Carlo comparison of missing $E_{T}$ is shown in Section 3 with the estimation of resolution and absolute scale from the data. The performance of the triggers is briefly discussed in Section 4. Finally, a summary is given in Section 5.

\section{Jet energy calibration and $p_{T}$ resolution}

CMS has developed a factorized multi-step procedure for the jet energy calibration (JEC) [10]. The following three subsequent corrections are devised to correct the jets to the corresponding 

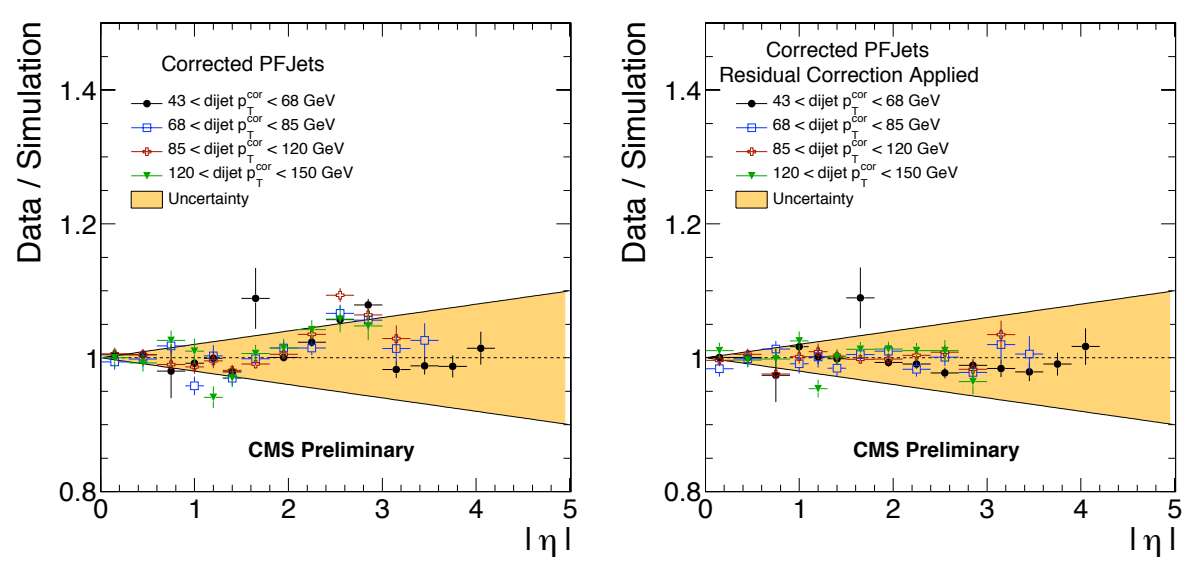

Figure 1: Data/MC ratio for relative response obtained from the dijet $p_{T}$ balance method for PFlow jets. On the left, the jets in data and MC are corrected for MC-truth JEC, and on the right, the jets in data are corrected for MC-truth JEC plus the residual correction. The $\pm 2 \%|\eta|$ band is overlaid. The few outlying points with large error bars are due to limited statistics on low- $p_{T}$ Monte Carlo samples. [2]

particle jet level: offset, relative and absolute corrections. The offset correction aims at correcting the jet energy for the unwanted excess energy due to electronics noise and pile-up. It is estimated from the data by measuring separately three components: noise, noise+one pile-up, and the total average offset $[2,11]$.

The relative correction removes variations in the jet response versus jet $\eta$ relative to a central control region. The jet response as a function of pseudorapidity is measured with the dijet $p_{T}$ balance technique $[2,12-15]$. The idea is to use $p_{T}$ balance in back-to-back dijet events with one jet (barrel jet) in the central control region of the calorimeter, $|\eta|<1.3$, and the other jet (probe jet) at arbitrary $\eta$. The data/MC ratio for the relative response is shown in Figure 1 for PFlow jets. In order to account the observed shift in data, an additional residual correction was derived on top of the nominal MC truth corrections, which is also shown in Figure 1.

The absolute correction removes variations in jet response versus jet $p_{T}$. The jet response as a function of jet $p_{T}$ is measured from $\gamma+$ jet events with $p_{T}$ balancing and MPF (missing $E_{T}$ projection fraction) methods. The $p_{T}$ balancing method $[13,14,16]$ exploits the balance in the transverse plane between the photon and the recoiling jet and uses the photon $p_{T}$, that is accurately measured in the crystal ECAL calorimeter, as the reference object. The MPF method [13] relies on the assumption that the $\gamma+$ jet events have no intrinsic missing $E_{T}$, and that the photon is perfectly balanced by the hadronic recoil in the transverse plane. The response of both methods for PFlow jets is shown in Figure 2. Further details on the jet energy calibrations are given in Ref. [2].

The jet $p_{T}$ resolutions are extracted from MC truth information from PYTHIA [17] QCD dijet MC events, simulated with full GEANT4 [18] simulation, and also measured directly from the data with the dijet asymmetry method $[19,20]$. The method exploits momentum conservation in the transverse plane of the dijet system, and is based (almost) exclusively on the measured kinematics of the dijet events. The obtained $p_{T}$ resolutions are shown in Figure 3 for the three jet types. The figure also shows results from PYTHIA QCD dijet sample obtained with the dijet asymmetry method, and the data/MC ratio. More details on the analysis are given in Ref. [2]. 

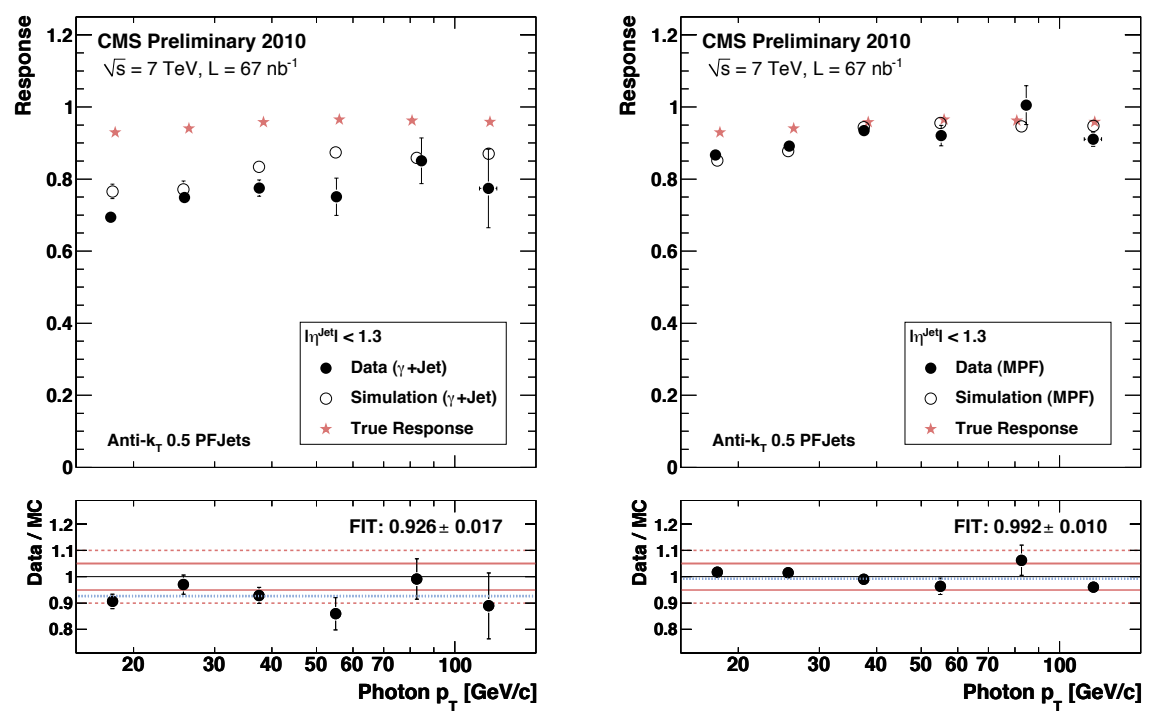

Figure 2: Response of $\left\langle p_{T} / p_{T}^{\gamma}\right\rangle$ versus $p_{T}^{\gamma}$ (left) and MPF response (right) in data and MC for PFlow jets. $\mathrm{MC}$ truth response is also shown. Data/MC ratio and the one-parameter linear fit function is shown at the bottom of the plots, together with $\pm 5 \%$ and $\pm 10 \%$ lines. [2]
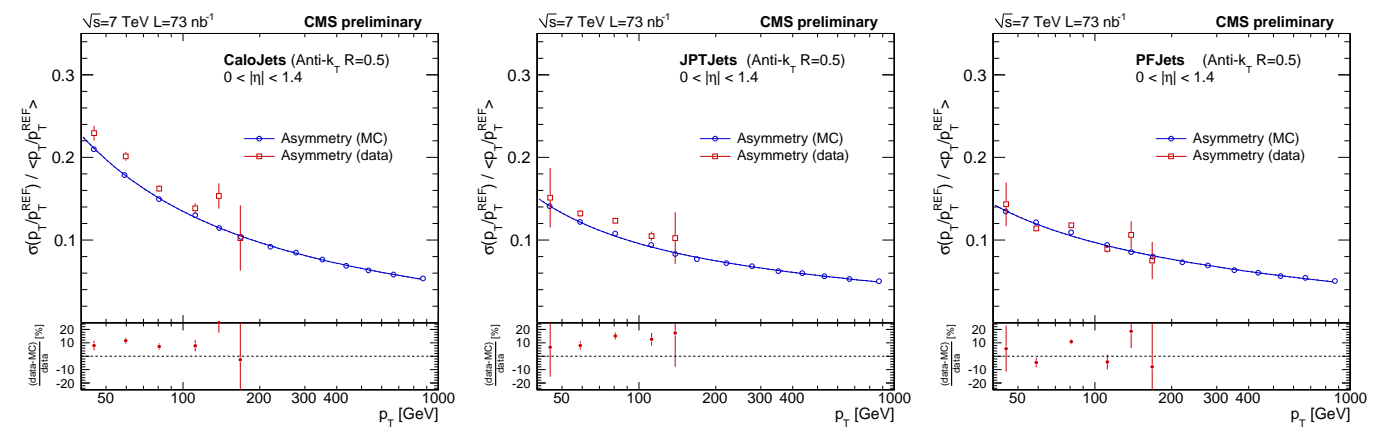

Figure 3: Calorimeter (left), JPT (middle), and PFlow (right) jet resolution for $0 \leq|\eta| \leq 1.4$ determined with the asymmetry method from QCD simulation and compared with the result from data using the same procedure. [2]

\section{Missing $E_{T}$ performance}

The distributions of the three $\mathbb{E}_{T}$ types and calorimeter $\sum E_{T}$ are shown in Figure 4 for events containing at least two jets with $p_{T}>25 \mathrm{GeV}$ and $|\eta|<3$. Comparisons between data and Monte Carlo show a reasonable agreement, especially for $\sum E_{T}$. In the case of $\mathbb{E}_{T}$, the Monte Carlo distribution is somewhat narrower. The observed differences are attributed to various sources including the imperfect modeling of the calorimeter response in the simulation. Further investigations have shown that the differences are most evident in the HCAL barrel and endcap regions, and the energy response in the endcap region is known to be underestimated in the simulation [21]. The analysis and results are further discussed in Refs. [5, 22].

The $\mathbb{E}_{T}$ resolution characterization is based on the $\sigma$ of a Gaussian fit to the $\mathbb{E}_{x, y}$ distribution 

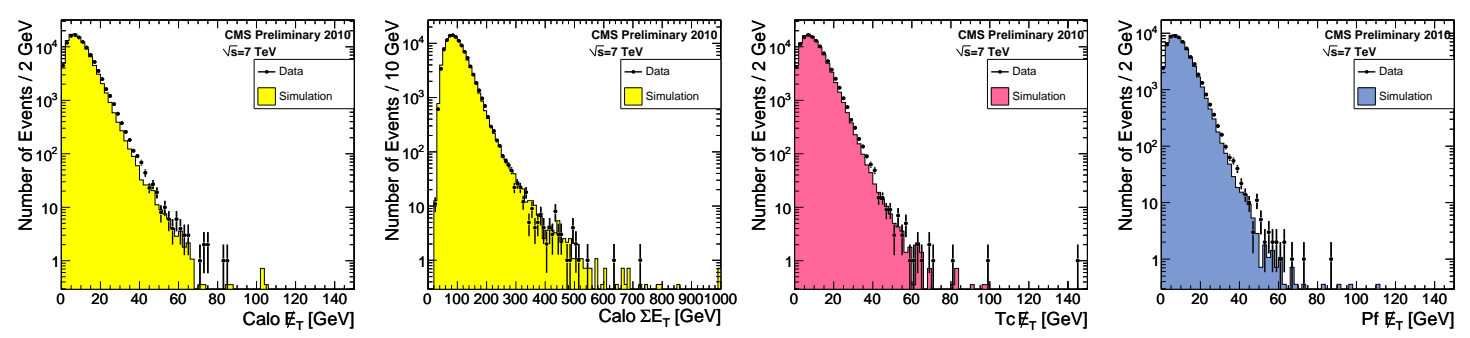

Figure 4: Calorimeter $\mathbb{E}_{T}$, calorimeter $\sum E_{T}$, track-corrected $\mathbb{E}_{T}$ and PFlow $\mathbb{E}_{T}$ distributions in inclusive dijet data $\left(p_{T}^{\text {jet } 1,2}>25 \mathrm{GeV}\right.$ and $\left|\eta^{\text {jet1,2 }}\right|<3$ ) compared with Monte Carlo Simulation. [22]

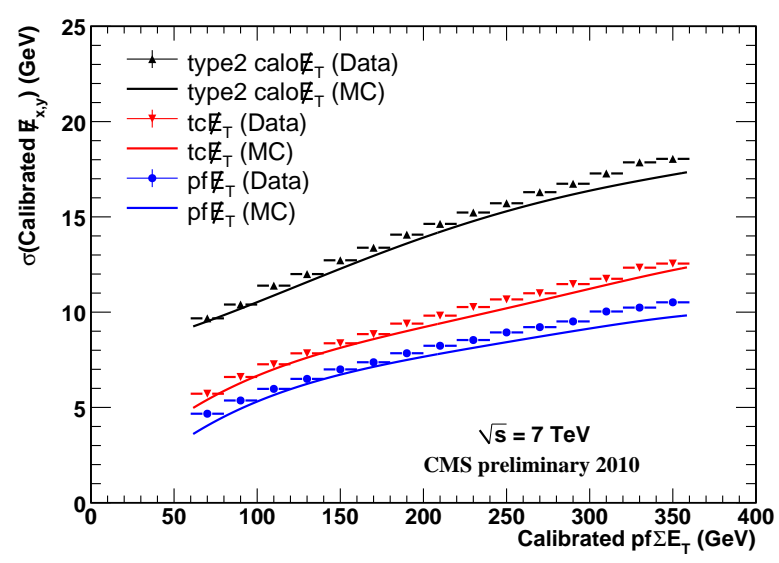

Figure 5: Calibrated $\mathbb{E}_{T}$ resolution versus calibrated pf $\sum E_{T}$ for the type-II corrected calorimeter $\mathbb{E}_{T}$, trackcorrected $\mathbb{E}_{T}$, and PFlow $\mathbb{E}_{T}$ in data and Monte Carlo samples. [22]

[8]. Because the $\mathbb{E}_{T}$ resolution has a strong dependence on the associated $\sum E_{T}$ it is presented as a function of $\sum E_{T}$. The resolutions of the three $\mathbb{E}_{T}$ reconstruction techniques are compared in Figure 5 in events containing at least two jets with $p_{T}>25 \mathrm{GeV}$. PFlow $\sum E_{T}$ is used for all $\mathbb{E}_{T}$ types because it is closest to the actual particle-level $\sum E_{T}$. In order to make a meaningful comparison, the measured $\mathbb{E}_{T}$ are calibrated to the same scale. The calibration procedure as well as further details of the analysis are given in Ref. [22]. Both track-corrected $\mathbb{E}_{T}$ and PFlow $\mathbb{E}_{T}$ show improvements in the $\mathbb{E}_{T}$ resolution compared to the calorimeter only $\mathbb{E}_{T}$, and the PFlow $\mathbb{E}_{T}$ yields the smallest $\mathbb{E}_{T}$ resolution.

The calibration scale of $\mathbb{E}_{T}$ measurement is studied in $\gamma+$ jet events, where the photon is detected and measured in ECAL with a good precision [23]. Since the hadronic system exhibits resolutions that are typically an order of magnitude larger in the $E_{T}$ ranges studied here, the photon serves effectively as a delta-function probe of the detector's response to the hadronic system. The mean value of scalar quantity $\left|\left\langle u_{\|}\right\rangle\right| / q_{T}$ measures the scale factor correction required for MET measurements in the class of events under study. Here $\vec{u}_{T}$ is the hadronic recoil, defined as the transverse momentum sum of all particles except the photon, $\vec{q}_{T}$ is the photon momentum in the transverse plane, and $u_{\|}=\vec{u}_{T} \cdot \vec{q}_{T} /\left|\vec{q}_{T}\right|$ is the parallel projection of the hadronic recoil onto the axis of the photon momentum.

The response curves extracted from data for the three $\mathbb{E}_{T}$ algorithms are shown in Figure 6. The 


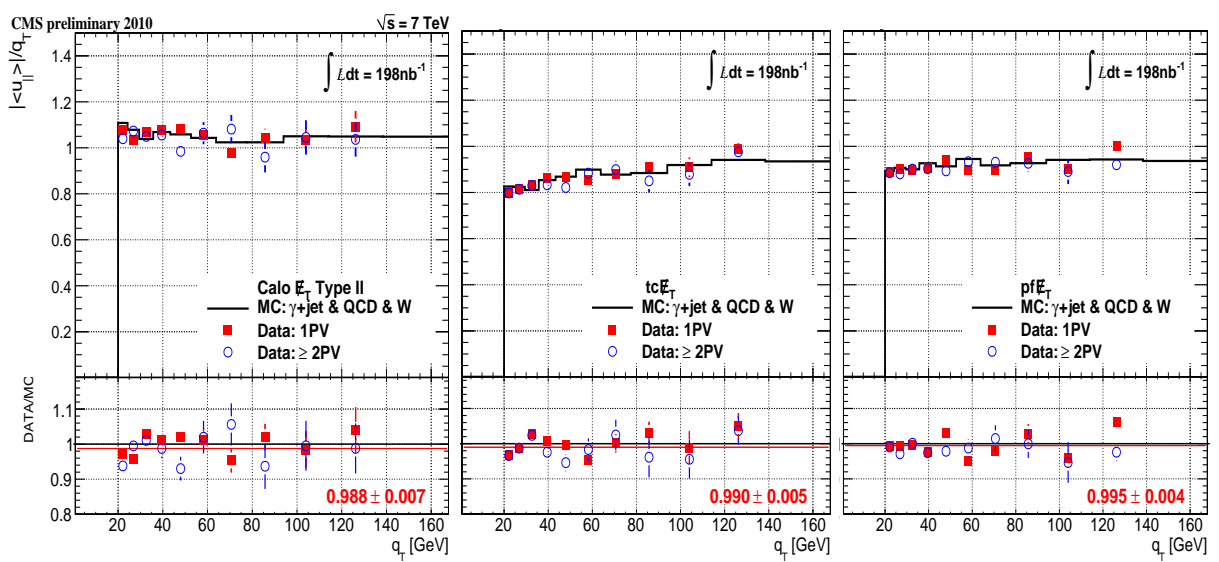

Figure 6: Response curves measured in photon+jet events, for calorimeter $\mathbb{E}_{T}$ (left), track-corrected $\mathbb{E}_{T}$ (middle), and PFlow $\mathbb{E}_{T}$ (right). Results are shown for events with exactly one primary vertex (full squares) and more than one (open circles). The upper frame of each figure shows the response in data (points) and Monte Carlo (histograms); the lower frame shows the ratio of data to Monte Carlo, with the brown line and the text at the bottom of the frame indicating the average data/MC ratio. The vertical axis labels at the far left apply to all three subfigures. [23]

agreement between data and Monte Carlo is good, and the results indicate that the three algorithms are distinct in their capabilities, performing differently in the recovery of hadronic activity in the detector. The higher than one response for calorimeter $\mathbb{E}_{T}$ is expected since the corresponding jet energy scale corrections are tuned for a mixture of quark and gluon jets, and the former are known to have a higher intrinsic response in the calorimeter with respect to latter, and are more common in the direct $\gamma$ production sample. The responses of track-corrected and PFlow $\mathbb{E}_{T}$ are underestimated due to the absence of jet energy corrections, which are not applied since the effects of non-linear response of the calorimeters are largely corrected by the algorithms themselves [23].

\section{Trigger performance}

The performance of triggers with the first data has been evaluated. The overall performance of jet, missing $E_{T}$, muon, and electron and photon triggers in both Level-1 and High Level Trigger is good, the plateaus are near $100 \%$, and the turn-on curves are steep.

\section{Summary}

The results of the jet energy calibration, jet $p_{T}$ resolution, and missing transverse energy resolution and calibration scale from the early $7 \mathrm{TeV} p p$ collisions at the CMS detector have been presented. For both jets and missing $E_{T}$, employing the tracking information improves the performance significantly compared to calorimeter-only information. The full Particle Flow technique is observed to give even smaller resolutions than simpler track-based corrections. The agreement between data and Monte Carlo is acceptable in the key quantities. The trigger performance is in general at a good level. 


\section{References}

[1] CMS Collaboration, The CMS experiment at the CERN LHC, JINST 3 (2008) S08004.

[2] CMS Collaboration, Jet Performance in pp Collisions at $\sqrt{s}=7 \mathrm{TeV}$, CMS PAS JME-10-003 (2010).

[3] CMS Collaboration, Jet Plus Tracks Algorithm for Calorimeter Jet Energy Corrections in CMS, CMS PAS JME-09-002 (2009).

[4] CMS Collaboration, Particle-Flow Event Reconstruction in CMS and Performance for Jets, Taus and $E_{\mathrm{T}}^{\mathrm{miss}}$, CMS PAS PFT-09-001 (2009).

[5] CMS Collaboration, Commissioning of the Particle-Flow Reconstruction in Minimum-Bias and Jet Events from pp Collisions at 7 TeV, CMS PAS PFT-10-002 (2010).

[6] CMS Collaboration, Commissioning of TrackJets in pp Collisions at $\sqrt{s}=7 \mathrm{TeV}, \mathrm{CMS}$ PAS JME-10-006 (2010).

[7] M. Cacciari, G. P. Salam, and G. Soyez, The anti-kt jet clustering algorithm, JHEP 04 (2008) 063.

[8] CMS Collaboration, Missing $E_{T}$ Performance in CMS, CMS PAS JME-01-001 (2007).

[9] CMS Collaboration, Track-corrected Missing Transverse Energy in CMS, CMS PAS JME-09-010 (2009).

[10] CMS Collaboration, Plans for Jet Energy Corrections at CMS, CMS PAS JME-07-002 (2007).

[11] CMS Collaboration, Offset Energy Correction for Cone Jets, CMS PAS JME-09-003 (2009).

[12] UA2 Collaboration, Measurement of Production and Properties of Jets at the CERN anti-p p Collider, Z. Phys. C20 (1983) 117.

[13] D0 Collaboration, Determination of the absolute jet energy scale in the Do calorimeters, Nucl. Inst. Meth. A 424 (1999) 352-394.

[14] CDF Collaboration, Determination of the jet energy scale at the Collider Detector at Fermilab, Nucl. Inst. Meth. A 566 (2006) 375-412.

[15] CMS Collaboration, Determination of Relative Jet Energy Scale at CMS from Dijet balance, CMS PAS JME-08-003 (2008).

[16] CMS Collaboration, Jet energy calibration with photon+jet events, CMS PAS JME-09-004 (2009).

[17] T. Sjostrand, S. Mrenna, and P. Skands, PYTHIA 6.4 physics and manual, JHEP 05 (2007) 026.

[18] S. Agostinelli et al., Geant4 - A simulation Toolkit, Nucl. Inst. Meth. A 506 (2003) 250-303

[19] CMS Collaboration, Measurement of the Jet Energy Resolutions and Jet Reconstruction Efficiency at CMS, CMS PAS JME-09-007 (2009).

[20] D0 Collaboration, High $p_{T}$ jets in p $\bar{p}$ collisions at $\sqrt{s}=630 \mathrm{GeV}$ and $1800 \mathrm{GeV}$, Phys.Rev.D 64 032003 (2000).

[21] CMS Collaboration, Single Particle Response in the CMS Calorimeters, CMS PAS JME-10-008 (2010).

[22] CMS Collaboration, Missing Transverse Energy Performance in Minimum-Bias and Jet Events from Proton-Proton Collisions at $\sqrt{s}=7$ TeV, CMS PAS JME-10-004 (2010).

[23] CMS Collaboration, CMS MET Performance in Events Containing Electroweak Bosons from pp Collisions at $\sqrt{s}=7 \mathrm{TeV}$, CMS PAS JME-10-005 (2010). . 